\title{
Characteristics of Veterans Accessing the Veterans Affairs Telephone Triage Who Have Depression or Suicidal Ideation: Opportunities for Intervention
}

\author{
Alison Ludwig*1,2, Cynthia Lucero-Obusan ${ }^{1}$, Patricia Schirmer ${ }^{1}$ and Mark Holodniy ${ }^{1,3}$ \\ 1VA Office of Public Health Surveillance and Research, Palo Alto, CA, USA; 'Epidemic Intelligence Service, Centers for Disease \\ Control and Prevention, Atlanta, GA, USA; ${ }^{3}$ Stanford University School of Medicine, Palo Alto, CA, USA
}

\section{Objective}

To characterize Veterans who call telephone triage because of suicidal ideation (SI) or depression and to identify opportunities for suicide prevention efforts among these telephone triage users using a biosurveillance application.

\section{Introduction}

Veterans accessing Veterans Affairs (VA) health care have higher suicide rates and more characteristics associated with suicide risk, including being male, having multiple medical and psychiatric comorbidities, and being an older age, compared with the general U.S. population. The Veterans Crisis Line is a telephone hotline available to Veterans with urgent mental health concerns; however, not all Veterans are aware of this resource. By contrast, telephone triage is a national telephone-based triage system used by the VA to assess and triage all Veterans with acute medical or mental health complaints.

\section{Methods}

The VA Electronic Surveillance System for Early Notification of Community-based Epidemics (ESSENCE) was queried for telephone triage calls during January 1-June 30, 2012. Calls were classified as SI or depression when the triage nurse selected SI or depression as the Veteran's chief complaint from a set of fixed options. Demographic and recommended follow-up time and location information was reviewed. A random sample of 20 SI calls and 50 depression calls were selected for chart review to determine whether Veterans were examined in a clinic or followed up by a clinician by telephone within 2 weeks of the veteran's call.

\section{Results}

During January 1-June 30, 2012, 253,573 total calls were placed to telephone triage. Among these calls, 2,460 unique Veterans placed 417 calls for SI and 2,290 calls for depression. This represents $1 \%$ $(2,707 / 253,573)$ of all calls placed during the period. All encounter information is available in the surveillance application within 24 hours of the call being placed. Median age of callers was 55 years (range: 19-94); 86\% were male; and 6\% placed repeat calls. The median number of repeat calls was 2 (range: $2-10$ ). Among the 2,707 calls for
SI or depression, $1,286(48 \%)$ were made after routine business hours (5:00 p.m.-8:00 a.m.), and 646 (24\%) were made on weekends. The greatest proportion of calls were from Wisconsin and Northern Illinois $(17 \%)$ and the Southeastern United States (14\%). Among the 2,290 calls for depression, 1,401 callers $(61 \%)$ were recommended for urgent follow up or within 24 hours. 771 (34\%) were assigned a follow up location of an emergency department; $117(5 \%)$ an urgent care; $1,332(58 \%)$ a physician's office or clinic; $52(2 \%)$ self-care at home; and $18(1 \%)$ were unspecified. Among the 417 calls for SI, callers $410(98 \%)$ were recommended for urgent follow-up or within 24 hours. $330(79 \%)$ were assigned a follow-up location of an emergency department; $38(9 \%)$ an urgent care; $43(10 \%)$ a physician's office or clinic; $3(1 \%)$ self-care at home; and $3(1 \%)$ unspecified. Among the 20 SI and 50 depression calls for which the charts were reviewed, $1(5 \%)$ SI call and $6(12 \%)$ depression calls had no documented follow-up by telephone or in person with a clinician within 2 weeks of initial call.

\section{Conclusions}

Telephone triage represents an additional data source available to surveillance applications. Although telephone triage is not the traditional method provided by the VA for triage of urgent mental health concerns, $>2,000$ Veterans called it with acute symptoms of SI or depression during January-June 2012. Training for suicide prevention should be prioritized for operators working during the high-volume periods of off-hours and weekends when approximately half and onequarter of calls were received, respectively. We recommend standard notification of suicide prevention coordinators regarding calls to telephone triage for SI or depression to prevent loss to follow-up among Veterans at risk for suicide. Further investigation into reasons for increased call burden in identified geographic areas also is recommended.

\section{Keywords}

Surveillance; Veterans; Suicide Risk

\section{*Alison Ludwig}

E-mail: Alison.Ludwig@va.gov 